Marcos Antonio Gaspar ${ }^{1}$

Edson Keyso de Miranda Kubo ${ }^{2}$

Daiane de Paula Leandro ${ }^{3}$

Keilla da Silva Santos ${ }^{4}$

Luciane Hiromi Nagai ${ }^{5}$

\title{
Qualidade de vida no trabalho para pessoas com deficiências: estudo de caso numa montadora de automóveis.
}

\section{Resumo}

A implantação da lei 8.213 (BRASIL, 1991) contribuiu significativamente para o aumento da participação de Pessoas com Deficiência (PCD) nas empresas e inseriu a questão da diversidade nos programas de qualidade de vida no trabalho (QVT). Porém, pouco se sabe sobre a efetividade dos programas de QVT. Este artigo se baseou em um estudo empírico em uma empresa multinacional automobilística situada na Região do ABC, com 720 PCDs. Os resultados da pesquisa mostram indícios de que a QVT é viável nas organizações e pode proporcionar a satisfação em PCDs sob o atual contexto da diversidade no ambiente de trabalho.

Palavras-chave: Qualidade de vida no trabalho. Pessoas com deficiências. Inclusão.

\section{Quality of working life for people with disabilities: a case study in a car manufacturer.}

\section{Abstract}

The implementation of law 8,213 (Brazil, 1991) has contributed significantly to increasing the participation of people with disabilities (PWD) in companies and revealed the diversity in the quality of working life (QWL) programs. However, little is known about the effectiveness of the QWL programs. To clarify this, this article is based on an empirical study in a automobile multinational company located in $A B C$ region, with 720 PWDs. The results show signs that the QWL is feasible in organizations and can provide the satisfaction in PWDs under the current context of diversity in working place.

Keywords: Quality of working life. People with disabilities. Inclusion.

\footnotetext{
1 Doutor em Administração pela USP; Professor da Fundação Salvador Arena e docente permanente do Programa de Pós-graduação (M/D) em Administração da Universidade Municipal de São Caetano do Sul. (marcos.gaspar@uscs.edu.br)

2 Doutor em Administração de Empresas pela Escola de Administração de Empresas de São Paulo da Fundação Getúlio Vargas (EAESP/FGV); Docente permanente do Programa de Pós-graduação em Administração (M/D) da Universidade Municipal de São Caetano do Sul (USCS). (edsonkubo@uscs.edu.br)

3 Tecnóloga em Processos Gerenciais; Fundação Salvador Arena - Faculdade de Tecnologia Termomecanica. (daianeh.com@gmail.com)

4 Tecnóloga em Processos Gerenciais; Fundação Salvador Arena - Faculdade de Tecnologia Termomecanica. (keilla.silva@gmail.com)

5 Tecnóloga em Processos Gerenciais; Fundação Salvador Arena - Faculdade de Tecnologia Termomecanica. (hi.nagai@hotmail.com)
} 


\section{Introdução}

A implantação da lei 8.213 (BRASIL, 1991) contribuiu significativamente para o aumento da participação de Pessoas com Deficiência (PCD) nas empresas e trouxe à tona a questão da diversidade sob o contexto dos programas de qualidade de vida no trabalho (QVT). Em essência, a diversidade diz respeito ao reconhecimento da individualidade e as formas como os indivíduos se identificam ou se percebem (FLEURY, 2000) em uma dada sociedade ou contexto organizacional. PCDs tendem a ser identificadas como minorias e há relatos de que foram historicamente estigmatizadas nas organizações (CENTERS e CENTERS, 1963; FARINA, SHERMAN e ALLEN, 1968). Observa-se aqui um contexto desafiador para as organizações que se propõem a lidar com a diversidade ao formularem seus programas de QVT, pois impactam na questão da auto-estima e da identidade dos PCDs.

Mesmo diante da obrigatoriedade de participação de PCDs imposta pela legislação, muitas empresas são adeptas a esta prática pelo atual contexto da gestão da diversidade. Desta forma, tais empresas se utilizam dessa prática também para se legitimarem perante os clientes (WAILES, MICHELSON, 2008), contratando pessoas com deficiência por perceberem a importância de promover a inclusão desses profissionais no mercado de trabalho.

Vale ressaltar que a QVT está intrinsecamente relacionada não somente à questão da diversidade, mas também a melhoria do desempenho organizacional (MORIN, 2005). Assim, para viabilizarem a gestão da diversidade e a melhoria do desempenho organizacional, as empresas devem se atentar à qualidade de vida proporcionada a esses profissionais (PCDs) em seu ambiente de trabalho. Para isso devem se preparar para oferecer um arranjo físico e ferramentas de trabalho adequadas para cada tipo de deficiência, além da criação de políticas específicas para tratarem do desenvolvimento desses profissionais. Não obstante, pouco se sabe a respeito da opinião de PCDs quanto à efetividade dos programas de QVT sob o contexto da diversidade.

Diante dos fatos explanados, este trabalho aborda a seguinte questão de pesquisa: Qual a opinião das PCDs e das Pessoas sem deficiência (PSD) quanto às ações de qualidade de vida no trabalho promovidas pela organização?

Para responder a essa pergunta de pesquisa, este artigo se baseou em um estudo empírico em uma empresa situada na região do $A B C$ com 13.480 colaboradores, dentre os quais 720 possuem algum tipo de deficiência. Os resultados da pesquisa mostram indícios de que o QVT é viável nas organizações e pode proporcionar efetivamente a satisfação em PCDs sob o atual contexto da diversidade.

Primeiramente o artigo apresentará um referencial teórico a respeito do contexto da qualidade de vida no trabalho, evidenciando o modelo clássico de Walton (1973). Posteriormente são apresentados a metodologia e os resultados da pesquisa, que serão discutidos para a elaboração das considerações finais.

\section{Qualidade de Vida no Trabalho}

Segundo Limongi-França (2001, p. 2), o conceito de qualidade de vida no trabalho (QVT) tem sido "analisado há tempos, estabilizando-se não como um modismo provisório e sim como um método que concretiza a busca do desenvolvimento humano e organizacional". Percebe-se que o termo qualidade na atmosfera empresarial já ganhou destaque. No primeiro momento, o termo qualidade era inserido em termos de produtos, serviços e processos e raramente era inserida a qualidade de vida do colaborador neste contexto. Porém, o conceito de qualidade atualmente deve ser expandido para abordar o indivíduo e sua vida organizacional (Freitas, 1999):

Não está sendo solicitado que as empresas abram mão de sua visão monetarizada de mundo, mas que elas honrem em ações o que costumam pregar em 
discursos que dizem que o ser humano é o principal "ativo". O ser humano, mortal e frágil, tem os seus defeitos, comete erros, mas quando ele é estimulado a substituir o coração por um chip ou uma maquina registradora, o mundo deve ter medo (FREITAS, 1999, p.7).

A citação de Freitas (1999) vai ao encontro das ideias de Walton (1973) ao afirmar que o conceito de QVT diz respeito ao atendimento de necessidades e aspirações humanas, calcado numa concepção de humanização e responsabilidade social da empresa. Isto implicaria em gerir de modo eficaz os fatores físicos, tecnológicos e sócio-pscicológicos que comprometem - clima organizacional e refletem no bem-estar do colaborador e na produtividade das companhias (ALVES; GALEÃO-SILVA, 2004; THOMPSON; NOEL, CAMPBELL, 2004).

Historicamente os funcionários eram submetidos a um ambiente de trabalho desprovido e insalubre, provenientes de ambientes sem iluminação, má ventilação, espaço insuficiente, entre outras características. Eram sobrecarregados por serviços pesados e não tinham autonomia suficiente para reivindicar seus direitos.

Tendo em vista estabelecer alguns parâmetros para regular às adequações das condições de trabalho relacionadas às características psicológicas dos colaboradores, o Ministério do Trabalho e Previdência Social instituiu a Portaria $n^{\circ} 3.751$, em 23/11/1990, que baixou a norma Regulamentadora $n^{\circ} 17$ (NR-17) que aborda designadamente a ergonomia. O intuito desta norma seria adequar todo o espaço físico da empresa, para que a mesma economize o máximo de movimentos necessários, reduzindo atividades repetitivas, melhoria no ritmo de produtividade e estabelecendo da melhor forma possível a minimização do empenho muscular do colaborador. Ruído, temperatura, iluminação, ergonomia, higiene, estresse, entre outros, são fatores que são analisados pelos próprios colaboradores por determinação da norma, e que devem convir para a concretização de ação de melhoria no ambiente físico da empresa e na precaução de doenças ocupacionais de seus empregados. Trata-se de uma norma voltada a minimizar efeitos psicossomáticos de um dado ambiente de trabalho sobre os funcionários. Esta consideração relacionada ao campo da psicossomática tem como prática promover a saúde, levando em consideração as funções ergonômicas e psicológicas.

A psicossomática preocupa-se com as interfaces, as conexões, as ligações entre o biológico, psicológico e social. Preocupa-se com a integração, ao partir do principio que o ser humano constitui-se numa unidade composta, indissociavelmente, por um corpo físico, uma subjetividade pessoal e singular, e um contexto histórico e social onde vive inserido. Cada ser humano é único, individual, mas todos os seres humanos vivem e convivem em interação uns com os outros e com o ambiente que os circunda (HULAK, 2003, p. 7).

Entre os distúrbios psicossomáticos do trabalho, o que ganha evidência de acordo com Limongi-França (1994, p.17) é o estresse, considerado pela autora como "o conjunto de reações que o organismo desenvolve ao ser submetido a uma situação que exige esforço para adaptarse." Desse modo, a exigência de esforço contínuo e a necessidade de adaptação constante por parte do indivíduo ao ambiente do trabalho refletem em fatores físicos e psicológicos que estão diretamente ligados à QVT. A revisão da literatura mostrou que o modelo clássico de QVT de Walton (1973) já levava em consideração esses fatores ao abordar o indivíduo e seu trabalho. Este modelo, focado nas percepções do indivíduo, contempla categorias que abrangem desde a remuneração, carreira, integração, o trabalho e suas garantias. Assim, a percepção do trabalhador quanto à significância do trabalho, o grau de responsabilidade pelos resultados e conhecimento real dos resultados obtidos, constituem aspectos fundamentais do modelo que é composto por oito fatores subdivididos em 24 dimensões, conforme detalhado a seguir: 
Figura 1 - Modelo de Qualidade de Vida no Trabalho

\begin{tabular}{|c|c|}
\hline Fatores de QVT & Dimensões \\
\hline A) Compensação justa e adequada & $\begin{array}{l}\text { 1. Renda (salário) adequada ao trabalho } \\
\text { 2. Equidade interna (compatibilidade interna) } \\
\text { 3. Equidade externa (compatibilidade externa) }\end{array}$ \\
\hline $\begin{array}{l}\text { B) Condições de segurança e saúde no } \\
\text { trabalho }\end{array}$ & $\begin{array}{l}\text { 4. Jornada de trabalho } \\
\text { 5. Ambiente físico }\end{array}$ \\
\hline $\begin{array}{l}\text { C) Utilização e desenvolvimento de } \\
\text { capacidades }\end{array}$ & $\begin{array}{l}\text { 6. Autonomia } \\
\text { 7. Significado da tarefa } \\
\text { 8. Identidade da tarefa } \\
\text { 9. Variedade de habilidades } \\
\text { 10. Retroação } \\
\end{array}$ \\
\hline $\begin{array}{l}\text { D) Oportunidades de crescimento e } \\
\text { segurança }\end{array}$ & $\begin{array}{l}\text { 11. Possibilidades de carreira } \\
\text { 12. Crescimento profissional } \\
\text { 13. Segurança do emprego }\end{array}$ \\
\hline E) Integração social na organização & $\begin{array}{l}\text { 14. Igualdade de oportunidades } \\
\text { 15. Relacionamentos interpessoais e grupais } \\
\text { 16. Senso comunitário }\end{array}$ \\
\hline F) Garantias constitucionais & $\begin{array}{l}\text { 17. Respeito às leis e direitos trabalhistas } \\
\text { 18. Privacidade pessoal } \\
\text { 19. Liberdade de expressão } \\
\text { 20. Normas e rotinas claras da organização }\end{array}$ \\
\hline G) Trabalho e espaço total de vida & 21. Papel balanceado do trabalho na vida pessoal \\
\hline H) Relevância social da vida no trabalho & $\begin{array}{l}\text { 22. Imagem da empresa } \\
\text { 23. Responsabilidade social pelos produtos / serviços } \\
\text { 24. Responsabilidade social pelos empregados }\end{array}$ \\
\hline
\end{tabular}

Fonte: Walton (1973).

Devido a sua influência enquanto modelo teórico para programas de QVT, os autores optaram por utilizá-lo neste artigo. Na próxima seção encontram-se a apresentação dos procedimentos metodológicos e explicações sobre o instrumento de pesquisa para verificar o programa de QVT em uma empresa brasileira.

\section{Metodologia da Pesquisa}

Conforme Marconi e Lakatos (2001) a metodologia de um trabalho científico deve abranger vários itens, que juntos deverão responder às questões: como, com quê, onde, quanto, entre outras. Com relação às pesquisas, é usual a classificação com base em seus objetivos gerais, desta forma é possível classificar as pesquisas em três grandes grupos: exploratórias, descritivas e explicativas (GIL, 2002). Esta é uma pesquisa descritiva de natureza quantitativa de caráter não probabilístico.

Na pesquisa descritiva, não há interferência do pesquisador, ele descreve o objeto de pesquisa. Para Gil (2002, p. 42) "são incluídas neste grupo as pesquisas que têm por objetivo levantar opiniões, atitudes e crenças de uma população".

O método quantitativo é muito utilizado no desenvolvimento das pesquisas descritivas, 
nas quais se procura identificar e classificar a relação entre variáveis.

A pesquisa de opinião se adéqua melhor aos fins requeridos no presente estudo, uma vez que, "procura saber atitudes, pontos de vista e preferências que as pessoas têm a respeito de algum assunto, com o objetivo de tomar decisões" (CERVO e BERVIAN, 2002, p. 67).

Utilizam-se os procedimentos da amostragem não-probabilística porque estes atendem de forma satisfatória aos objetivos de amostragem, possuem custo e tempo menor. Além disso, em alguns casos "a amostragem não-probabilística seja a única alternativa viável. A população total pode não estar disponível para estudo em certos casos" (COOPER e SHINDLER, 2003, p.167).

Assim, como pesquisa de campo, este estudo empírico investiga a opinião da qualidade de vida no trabalho dos trabalhadores, efetuando uma análise comparativa entre os resultados das pessoas com e sem deficiência, a fim de constatar se as políticas adotadas geram os mesmos resultados na opinião de ambos os grupos.

O estudo empírico foi realizado em uma empresa presente há mais de 50 anos no país, situada na região do $\mathrm{ABC}$. A escolha da empresa foi fundamentada pelo conhecimento de sua preocupação com a aplicação da responsabilidade social em todas as suas ações, princípio este que está presente inclusive em seu código de ética e missão.

Outro fator favorável se deve ao fato da empresa realizar a inclusão de PCD em seu quadro colaborativo, inclusive acima do que é exigido pela lei federal $n^{\circ} 8.213$. Hoje a empresa conta com 13.480 colaboradores, dentre os quais 720 possuem algum tipo de deficiência, representando assim 5,3\% deste total, ou seja, 46 colaboradores a mais do que é exigido por lei.

Pelo fato de a população total (720 colaboradores com deficiência), não estar disponível para o estudo e uma vez que o intuito da pesquisa é identificar a opinião de colaboradores com e sem deficiência, optou-se pela amostragem não-probabilística.

Para a coleta dos dados foi selecionado um grupo representativo de $4,2 \%$ do total de PCD, 15 indivíduos e, para análise comparativa foi selecionado a mesma quantidade de pessoas sem deficiência (PSD), observando-se as mesmas áreas/cargos, perfazendo assim um total de 30 colaboradores respondentes.

A amostragem por acessibilidade e conveniência se tornou interessante para o estudo, pois demonstrou-se mais viável aos autores. Ela é usada para se ter ideia sobre um assunto de interesse, e às vezes, os resultados obtidos são tão positivos, que se torna desnecessário a utilização de amostras mais sofisticadas (COOPER; SCHINDLER, 2003).

Como instrumento de pesquisa, utilizou-se um protocolo padronizado conhecido por medidas de opinião e atitude que, segundo Marconi e Lakatos (2001), é o mais apropriado quando o que se deseja é o levantamento de diferentes opiniões e atitudes sobre determinado assunto, para compará-los posteriormente.

Após definido o tipo de instrumento de pesquisa, foram elaboradas assertivas utilizando uma escala tipo Likert com cinco pontos, com base no modelo de QVT desenvolvido por Walton (1973). A escala Likert de cinco pontos utilizada em cada uma das 24 assertivas do instrumento de pesquisa contemplava as seguintes opções: DT - discordo totalmente, D discordo, I - imparcial, C - concordo e, por fim, CT - concordo totalmente. Posteriormente, a gradação exposta pela escala empregada nas assertivas foi transformada em pontuação, de modo a melhor comparar os resultados apresentados por ambos os grupos analisados na pesquisa de campo. A pontuação adotada foi: DT (1 ponto), D (2 pontos), I (3 pontos), C (4 pontos) e CT (5 pontos).

O modelo de Walton (1973) possui oito fatores sendo eles: a) compensação justa e adequada, b) condições de segurança e saúde no trabalho, c) utilização e desenvolvimento 
de capacidades, d) oportunidades de crescimento e segurança, e) integração social na organização, f) garantias constitucionais, g) trabalho e espaço total de vida, h) relevância social da vida no trabalho. Como desdobramento desses oito fatores há 24 dimensões representadas por 24 assertivas disponíveis no instrumento de pesquisa.

\section{Apresentação dos Resultados}

A observação direta intensiva e levantamento de informações junto ao departamento de recursos humanos da empresa mostraram que a empresa possui um programa bem estruturado para obtenção de inclusão e qualidade de vida para PCD, chamado de: PROGRAMA INCLUSÃO EFICIENTE - CINCO ESTRELAS que contempla o cumprimento do Termo de Ajuste de Conduta (TAC) - onde todas as empresas possuem um número determinado por lei (Lei 8213, Brasil, 1991), de PCD para fazer parte do quadro de colaboradores, que na situação atual da organização é $5 \%$ dos colaboradores efetivos. Hoje, essa é uma etapa cumprida, uma vez que a empresa conta em seu quadro de colaboradores com deficiência mais do que é determinado por lei, chegando a 720 PCD, quando o obrigatório é 674 . Isso é possível, pois a etapa de contratação é realizada com sustentabilidade, ou seja, a cada 20 contratações de pessoas sem deficiência, é contratado um PCD.

Assim, a pesquisa se preocupou em responder se existe equidade dessas ações na opinião das PCD e na opinião das que não possuem tais particularidades. As respostas foram obtidas através do instrumento de pesquisa de padronização conhecido por medidas de opinião e atitude. Foram consultados 30 colaboradores operacionais, sendo quinze com algum tipo de deficiência e quinze sem deficiência. Os resultados serão explanados a seguir através das respostas dos funcionários diante das assertivas geradas a partir do modelo de Walton (1973). Diante do volume de assertivas e respostas, optou-se por apresentar as mais representativas dentro de cada categoria, deixando para a seção de considerações finais a interpretação definitiva da visão geral dos dados coletados na pesquisa de campo:

a) Em um primeiro momento observou-se o grau de satisfação do colaborador com sua remuneração em relação à sua prestação de serviço. A partir dessa dimensão foi elaborada a seguinte assertiva: O meu salário é justo de acordo com as atividades que eu faço no meu trabalho.

Tabela 1 - Resultados de Pesquisa - Compensação Justa e Adequada - Renda adequada ao trabalho

\begin{tabular}{|c|c|c|c|c|}
\cline { 2 - 5 } \multicolumn{1}{c|}{} & \multicolumn{4}{c|}{ COMPESAÇÃo JUSTA E ADEQUADA - Renda (salário) adequada ao trabalho } \\
\cline { 2 - 5 } \multicolumn{1}{c|}{} & \multicolumn{4}{c|}{ 1. O meu salário é justo de acordo com as atividades que eu faço no meu trabalho } \\
\cline { 2 - 6 } & Pessoa com Deficiência (PCD) & \multicolumn{2}{|c|}{ Pessoa sem deficiência (PSD) } \\
\cline { 2 - 5 } D.T. & Quantidade & Porcentagem (\%) & Quantidade & Porcentagem (\%) \\
D. & 1 & $0 \%$ & 1 & $7 \%$ \\
I. & 4 & $6 \%$ & 2 & $13 \%$ \\
C. & 7 & $27 \%$ & 0 & $0 \%$ \\
C.T. & 3 & $47 \%$ & 9 & $60 \%$ \\
\hline TOTAL & 15 & $20 \%$ & 3 & $20 \%$ \\
\hline
\end{tabular}

Fonte: elaborado pelos autores 
Como resultado, a opinião das pessoas sem deficiência foi mais positiva do que a das PCD, onde das 30 pessoas consultadas, $80 \%$ das PSD e $67 \%$ das PCD concordaram com a assertiva.

b) Com o intuito de mensurar a adequação da carga horária da jornada de trabalho, para não prejudicar a saúde do colaborador. Solicitou-se que os respondentes analisassem a seguinte assertiva: A minha jornada de trabalho é adequada, não prejudicando a minha saúde.

Tabela 2 - Resultados de Pesquisa - Condições de Segurança e Saúde de Trabalho - Jornada de Trabalho

\begin{tabular}{|c|c|c|c|c|}
\cline { 2 - 5 } \multicolumn{1}{c|}{} & \multicolumn{4}{|c|}{ CONDIÇõES DE SEGURANÇA E SAÚDE DE TRABALHO - Jornada de Trabalho } \\
\cline { 2 - 5 } \multicolumn{1}{c|}{} & \multicolumn{2}{|c|}{ 4. A minha jornada de trabalho adequada, não predujicando a minha saúde } \\
\cline { 2 - 5 } & Pessoa com Deficiência (PCD) & \multicolumn{2}{|c|}{ Pessoa sem deficiência (PSD) } \\
\cline { 2 - 5 } D.T. & 0 & $0 \%$ & 0 & $0 \%$ \\
D. & 0 & $0 \%$ & 0 & $0 \%$ \\
I. & 3 & $20 \%$ & 2 & $13 \%$ \\
C. & 10 & $67 \%$ & 9 & $60 \%$ \\
C.T. & 2 & $13 \%$ & 4 & $27 \%$ \\
\hline TOTAL & 15 & $100 \%$ & 15 & $100 \%$ \\
\hline
\end{tabular}

Fonte: elaborado pelos autores.

O resultado foi positivo, pois nenhum colaborador discordou com a assertiva, visto que se trata de um item muito importante por estar diretamente relacionado à saúde do colaborador. $87 \%$ das PSD e $80 \%$ das PCD concordaram com a assertiva.

c)Já em relação ao ambiente físico do trabalho, apresentou-se a seguinte assertiva: O ambiente físico da minha área é seguro e apropriado. 
Tabela 3 - Resultados de Pesquisa - Condições de Segurança e Saúde de Trabalho - Ambiente Físico

\begin{tabular}{|c|c|c|c|c|}
\cline { 2 - 5 } \multicolumn{1}{c|}{} & \multicolumn{4}{|c|}{ CONDIÇõES DE SEGURANÇA E SAÚDE DE TRABALHO - Ambiente Físico } \\
\cline { 2 - 5 } \multicolumn{1}{c|}{} & \multicolumn{3}{c|}{5.0 ambiente fisico da minha área é seguro e apropriado. } \\
\cline { 2 - 5 } & Pessoa com Deficiência (PCD) & \multicolumn{2}{|c|}{ Pessoa sem deficiência (PSD) } \\
\cline { 2 - 5 } D.T. & 0 & $0 \%$ & 0 & $0 \%$ \\
D. & 0 & $0 \%$ & 1 & $7 \%$ \\
I. & 4 & $26 \%$ & 2 & $13 \%$ \\
C. & 10 & $67 \%$ & 11 & $73 \%$ \\
C.T. & 1 & $7 \%$ & 1 & $7 \%$ \\
\hline TOTAL & 15 & $100 \%$ & 15 & $100 \%$ \\
\hline
\end{tabular}

Fonte: elaborado pelos autores.

Nesta assertiva foi constatada similaridade entre as opiniões, concentrando a maioria das respostas no grau de concordância. Porém, entre os colaboradores sem deficiência foi constatado uma discordância, o que deve ser considerado e avaliado posteriormente pela empresa, uma vez que se trata de um item de suma importância.

d) No que tange a autonomia do colaborador em sua área de atuação, torna-se importante relevante identificar se existe preocupação em proporcionar, principalmente às PCD, um ambiente adequado para a execução de tarefas compatíveis às possíveis deficiências. Relacionado à autonomia, a assertiva elaborada foi: A empresa me dá liberdade e autonomia nas minhas atividades na área em que atuo.

Tabela 4 - Resultado de Pesquisa - Utilização e Desenvolvimento de Capacidades - Autonomia

\begin{tabular}{|c|c|c|c|c|}
\hline & \multicolumn{4}{|c|}{ UTILIZAÇÃO E DESENVOLVIMENTO DE CAPACIDADES - Autonomia } \\
\hline & \multicolumn{4}{|c|}{ 6. A empresa me dá liberdade e autonomia nas minhas atividides na área em que atuo. } \\
\hline & \multicolumn{2}{|c|}{ Pessoa com Deficiência (PCD) } & \multicolumn{2}{|c|}{ Pessoa sem deficiência (PSD) } \\
\hline & Quantidade & Porcentagem (\%) & Quantidade & Porcentagem (\%) \\
\hline D.T. & 0 & $0 \%$ & 0 & $0 \%$ \\
\hline D. & 0 & $0 \%$ & 2 & $13 \%$ \\
\hline I. & 5 & $33 \%$ & 2 & $13 \%$ \\
\hline c. & 9 & $60 \%$ & 10 & $67 \%$ \\
\hline C.T. & 1 & $7 \%$ & 1 & $7 \%$ \\
\hline TOTAL & 15 & $100 \%$ & 15 & $100 \%$ \\
\hline
\end{tabular}

Fonte: elaborado pelos autores. 
O resultando foi positivo para ambos os grupos, surpreendentemente obtendo duas discordâncias entre as PSD, o que seria mais provável acontecer com as PCD devido suas particularidades.

e) Em relação às tarefas, observou-se a seguinte opinião dos respondentes frente à assertiva: Eu considero as atividades que faço no meu trabalho muito importante.

Tabela 5-Resultado de Pesquisa - Utilização e Desenvolvimento de Capacidades - Significado da Tarefa

\begin{tabular}{|c|c|c|c|c|}
\cline { 2 - 5 } \multicolumn{1}{c|}{} & \multicolumn{4}{|c|}{ UTILIZAÇÃO E DESENVOLVIMENTO DE CAPACIDADES - Significado da Tarefa } \\
\cline { 2 - 5 } \multicolumn{1}{c|}{} & \multicolumn{2}{c|}{ 7. Eu considero as atividades que faço no trabalho muito importantes } \\
\cline { 2 - 5 } & \multicolumn{2}{|c|}{ Pessoa com Deficiência (PCD) } & \multicolumn{2}{c|}{ Pessoa sem deficiência (PSD) } \\
\cline { 2 - 5 } D.T. & 0 & $0 \%$ & 0 & $0 \%$ \\
D. & 0 & $0 \%$ & 0 & $0 \%$ \\
I. & 3 & $20 \%$ & 3 & $20 \%$ \\
C. & 9 & $60 \%$ & 9 & $60 \%$ \\
C.T. & 3 & $20 \%$ & 3 & $20 \%$ \\
\hline TOTAL & 15 & $100 \%$ & 15 & $100 \%$ \\
\hline
\end{tabular}

Fonte: elaborado pelos autores.

O resultado das opiniões para essa assertiva foi idêntico, onde $80 \%$ dos colaboradores de cada grupo consideraram a assertiva compatível com suas realidades.

f) Quando indagados a respeito da identidade quanto às tarefas que desempenho através da assertiva: Sinto-me muito à vontade ao desempenhar as minhas atividades, os respondentes se manifestaram do seguinte modo:

Tabela 6 - Resultado de Pesquisa - Utilização e Desenvolvimento de Capacidades - Identidade da tarefa

\begin{tabular}{|c|c|c|c|c|}
\cline { 2 - 5 } \multicolumn{1}{c|}{} & \multicolumn{4}{|c|}{ UTILIZAÇãO E DESENVOLVIMENTO DE CAPACIDADES - Identidade da Tarefa } \\
\cline { 2 - 5 } \multicolumn{1}{c|}{} & \multicolumn{3}{c|}{ 8. Sinto-me muito à vontade ao desempenhar as minhas atividades } \\
\cline { 2 - 5 } \multicolumn{1}{c|}{ Pessoa com Deficiência (PCD) } & \multicolumn{2}{c|}{ Pessoa sem deficiência (PSD) } \\
\cline { 2 - 5 } D.T. & Quantidade & Porcentagem (\%) & Quantidade & Porcentagem (\%) \\
D. & 0 & $0 \%$ & 0 & $0 \%$ \\
I. & 1 & $0 \%$ & 0 & $0 \%$ \\
C. & 10 & $7 \%$ & 2 & $13 \%$ \\
C.T. & 4 & $66 \%$ & 11 & $74 \%$ \\
\hline TOTAL & 15 & $27 \%$ & 2 & $13 \%$ \\
\hline
\end{tabular}

Fonte: elaborado pelos autores. 
Nesta assertiva o resultado geral das PCD foi sutilmente superior ao das PSD, onde $93 \%$ e $87 \%$, respectivamente, concordaram com a assertiva.

g) Em relação à retroação, a assertiva elaborada foi: Sempre que sinto necessidade, tenho a oportunidade e liberdade de expressas minha opinião sobre os resultados das minhas atividades.

Tabela 7 - Resultado de Pesquisa - Utilização e Desenvolvimento de Capacidades - Retroação e Retroinformação

\begin{tabular}{|c|c|c|c|c|}
\cline { 2 - 5 } \multicolumn{1}{c|}{} & \multicolumn{4}{|c|}{ UTILIZAÇÃO E DESENVOLVIMENTO DE CAPACIDADES - Retroação e Retroinformação } \\
\cline { 2 - 5 } & \multicolumn{1}{|c|}{$\begin{array}{r}\text { 10. Sempre que sinto necessidade, tenho a oportunidade e liberdade de expressar minha } \\
\text { opinião sobre os resultados das minhas atividades }\end{array}$} \\
\cline { 2 - 5 } & Quantidade & Porcentagem (\%) & Quantidade & Porcentagem (\%) \\
\cline { 2 - 5 } D.T. & 0 & $0 \%$ & 0 & $0 \%$ \\
I. & 2 & $13 \%$ & 3 & $20 \%$ \\
C. & 2 & $13 \%$ & 2 & $14 \%$ \\
C.T. & 9 & $61 \%$ & 8 & $53 \%$ \\
\hline TOTAL & 15 & $13 \%$ & 2 & $13 \%$ \\
\hline
\end{tabular}

Fonte: elaborado pelos autores.

Os resultados foram próximos para ambos os grupos, mas deve-se atentar para a porcentagem que discordam da assertiva, onde $20 \%$ das PSD e $13 \%$ das PCD não percebem esta afirmativa como uma realidade em seu ambiente de trabalho.

h) Relacionado à oportunidade elaborou-se a seguinte assertiva: $A$ empresa tem um plano de carreira definido para mim.

Tabela 8 - Resultado de Pesquisa - Oportunidade de Crescimento e Segurança - Possibilidade de Carreira

\begin{tabular}{|c|c|c|c|c|}
\hline & \multicolumn{4}{|c|}{ OPORTUNIDADE DE CRESCIMENTO E SEGURANÇA - Possibilidades de Carreira } \\
\hline & \multicolumn{4}{|c|}{ 11. A empresa tem um plano de carreira definido para mim } \\
\hline & \multicolumn{2}{|c|}{ Pessoa com Deficiência (PCD) } & \multicolumn{2}{|c|}{ Pessoa sem deficiência (PSD) } \\
\hline & Quantidade & Porcentagem $(\%)$ & Quantidade & Porcentagem $(\%)$ \\
\hline D.T. & 0 & $0 \%$ & 7 & $47 \%$ \\
\hline D. & 5 & $33 \%$ & 2 & $13 \%$ \\
\hline I. & 4 & $27 \%$ & 4 & $27 \%$ \\
\hline C. & 5 & $33 \%$ & 2 & $13 \%$ \\
\hline C.T. & 1 & $7 \%$ & 0 & $0 \%$ \\
\hline TOTAL & 15 & $100 \%$ & 15 & $100 \%$ \\
\hline
\end{tabular}

Fonte: elaborado pelos autores. 
Nesta dimensão os resultados não foram muito positivos para ambos os grupos, mas percebe-se que para as PSD o grau de percepção de um plano de carreira é menor do que para as PCD, onde $60 \%$ e $33 \%$ dos colaboradores, respectivamente, não concordam com esta assertiva.

i) No que tange à estabilidade no emprego, os funcionários responderam à seguinte assertiva: Eu me sinto seguro e estável em meu emprego.

Tabela 9 - Resultado de Pesquisa - Oportunidade de Crescimento e Segurança - Segurança no Emprego

\begin{tabular}{|c|c|c|c|c|}
\cline { 2 - 5 } \multicolumn{1}{c|}{} & \multicolumn{4}{c|}{ OPORTUNIDADE DE CRESCIMENTO E SEGURANÇA - Segurança no Emprego } \\
\cline { 2 - 5 } \multicolumn{1}{c|}{} & \multicolumn{4}{c|}{ 13. Eu me sinto seguro estável em meu emprego } \\
\cline { 2 - 5 } & Pessoa com Deficiência (PCD) & \multicolumn{2}{c|}{ Pessoa sem deficiência (PSD) } \\
\cline { 2 - 5 } D.T. & 0 & $0 \%$ & 0 & $0 \%$ \\
D. & 1 & $7 \%$ & 1 & $7 \%$ \\
I. & 0 & $0 \%$ & 1 & $7 \%$ \\
C. & 12 & $80 \%$ & 9 & $60 \%$ \\
C.T. & 2 & $13 \%$ & 4 & $26 \%$ \\
\hline TOTAL & 15 & $100 \%$ & 15 & $100 \%$ \\
\hline
\end{tabular}

Fonte: elaborado pelos autores.

O resultado foi positivo e próximo para ambos os grupos. Onde $86 \%$ das PSD e $93 \%$ das PCD concordam com a assertiva e apenas um colaborador de cada grupo discorda.

j) Em relação ao ambiente solidário e à integração social na organização, os respondentes foram submetidos à seguinte afirmação: Eu me sinto parte da empresa onde todos são solidários.

Tabela 10 - Resultado de Pesquisa - Integração Social na Organização - Senso Comunitário

\begin{tabular}{|c|c|c|c|c|}
\cline { 2 - 5 } \multicolumn{1}{c|}{} & \multicolumn{4}{|c|}{ INTEGRAÇÃO SOCIAL NA ORGANIZAÇÃO - Senso Comunitário } \\
\cline { 2 - 5 } & \multicolumn{4}{|c|}{ 16.Eu me sinto parte desta empresa onde são todos solidários } \\
\cline { 2 - 6 } & \multicolumn{2}{|c|}{ Pessoa com Deficiência (PCD) } & \multicolumn{2}{|c|}{ Pessoa sem deficiência (PSD) } \\
\cline { 2 - 6 } D.T. & 0 & $0 \%$ & 1 & $7 \%$ \\
D. & 1 & $7 \%$ & 2 & $13 \%$ \\
I. & 5 & $33 \%$ & 3 & $20 \%$ \\
C. & 7 & $47 \%$ & 8 & $53 \%$ \\
C.T. & 2 & $13 \%$ & 1 & $7 \%$ \\
\hline TOTAL & 15 & $100 \%$ & 15 & $100 \%$ \\
\hline
\end{tabular}

Fonte: elaborado pelos autores. 
O resultado foi parecido e positivo para os dois grupos, com $60 \%$ de concordância para ambos.

k) Em relação à garantias constitucionais e direitos perguntou-se: $A$ empresa cumpre totalmente as leis e direitos trabalhistas.

Tabela 11 - Resultado de Pesquisa - Garantias Constitucionais - Respeito às leis e direitos trabalhistas

\begin{tabular}{|c|c|c|c|c|}
\cline { 2 - 5 } \multicolumn{1}{c|}{} & \multicolumn{4}{c|}{ GARANTIAS CONSTITUCIONAIS - Respeito às leis e direitos trabalhistas } \\
\cline { 2 - 5 } \multicolumn{1}{c|}{} & \multicolumn{3}{c|}{ 17. A empresa cumpre totalmente as leis e direitos trabalhistas. } \\
\cline { 2 - 5 } \multicolumn{1}{c|}{} & Pessoa com Deficiência (PCD) & \multicolumn{2}{|c|}{ Pessoa sem deficiência (PSD) } \\
\cline { 2 - 5 } D.T. & 0 & $0 \%$ & 0 & $0 \%$ \\
D. & 0 & $0 \%$ & 0 & $0 \%$ \\
I. & 2 & $13 \%$ & 1 & $7 \%$ \\
C. & 6 & $40 \%$ & 9 & $60 \%$ \\
C.T. & 7 & $47 \%$ & 5 & $33 \%$ \\
\hline TOTAL & 15 & $100 \%$ & 15 & $100 \%$ \\
\hline
\end{tabular}

Fonte: elaborado pelos autores.

Os resultados foram positivos, não tendo nenhuma discordância quanto ao tema. Tendo uma sutil maioria entre as PSD que concordam com a assertiva.

I) Quanto à dimensão de respeito ao espaço individual de cada funcionário, questionou-se o respeito à privacidade através da assertiva: $A$ empresa respeita minha privacidade pessoal.

Tabela 12 - Resultado de Pesquisa - Garantias Constitucionais - Privacidade Pessoal

\begin{tabular}{|c|c|c|c|c|}
\cline { 2 - 5 } \multicolumn{1}{c|}{} & \multicolumn{4}{c|}{ GARANTIAS CONSTITUCIONAIS - Privacidade Pessoal } \\
\cline { 2 - 5 } \multicolumn{1}{c|}{} & \multicolumn{4}{c|}{ 18. A empresa respeita minha provacidade pessoal } \\
\cline { 2 - 5 } \multicolumn{1}{c|}{} & Pessoa com Deficiência (PCD) & Pessoa sem deficiência (PSD) \\
\cline { 2 - 5 } D.T. & 0 & $0 \%$ & 0 & $0 \%$ \\
D. & 0 & $0 \%$ & 0 & $0 \%$ \\
I. & 0 & $0 \%$ & 2 & $13 \%$ \\
C. & 11 & $73 \%$ & 10 & $67 \%$ \\
C.T. & 4 & $27 \%$ & 3 & $20 \%$ \\
\hline TOTAL & 15 & $100 \%$ & 15 & $100 \%$ \\
\hline
\end{tabular}

Fonte: elaborado pelos autores. 
O resultado também foi positivo, não tendo ninguém que discorde desta afirmativa. Sendo melhor para as PCD, uma vez que $100 \%$ dos colaboradores deste grupo concordam com a assertiva.

m) Em relação ao trabalho e a vida pessoal foi elaborada a seguinte assertiva:

Eu consigo conciliar o meu trabalho e a minha vida pessoal.

Tabela 13 - Resultado de Pesquisa - Trabalho e Espaço Total de Vida - Papel balanceado do trabalho na vida pessoal

\begin{tabular}{|c|c|c|c|c|}
\hline & \multirow{2}{*}{\multicolumn{4}{|c|}{$\begin{array}{c}\text { TRABALHO E ESPAÇO TOTAL DE VIDA - Papel balanceado do trabalho na vida pessoa } \\
\text { 21. Eu consigo conciliar meu trabalho e a minha vida pessoal }\end{array}$}} \\
\hline & & & & \\
\hline & \multicolumn{2}{|c|}{ Pessoa com Deficiência (PCD) } & \multicolumn{2}{|c|}{ Pessoa sem deficiência (PSD) } \\
\hline & Quantidade & Porcentagem (\%) & Quantidade & Porcentagem (\%) \\
\hline D.T. & 0 & $0 \%$ & 0 & $0 \%$ \\
\hline D. & 0 & $0 \%$ & 0 & $0 \%$ \\
\hline I. & 0 & $0 \%$ & 1 & $7 \%$ \\
\hline c. & 9 & $60 \%$ & 10 & $67 \%$ \\
\hline С.T. & 6 & $40 \%$ & 4 & $26 \%$ \\
\hline TOTAL & 15 & $100 \%$ & 15 & $100 \%$ \\
\hline
\end{tabular}

Fonte: elaborado pelos autores.

O resultado foi consideravelmente positivo, não sendo apontando nenhuma discordância com o tema para ambos os grupos.

n) Em relação à satisfação no trabalho: Eu tenho satisfação em dizer que trabalho nesta empresa quando me perguntam.

Tabela 14 - Resultado de Pesquisa - Relevância Social da Vida no Trabalho - Imagem da Empresa

\begin{tabular}{|c|c|c|c|c|}
\cline { 2 - 5 } \multicolumn{1}{c|}{} & \multicolumn{4}{|c|}{ RELEVÂNCIA SOCIAL DA VIDA NO TRABALHO - Imagem da Empresa } \\
\cline { 2 - 5 } \multicolumn{1}{c|}{} & \multicolumn{2}{|c|}{ 22. Eu tenho satisfação em dizer que trabalho nesta empresa quando me perguntam } \\
\cline { 2 - 5 } & Pessoa com Deficiência (PCD) & \multicolumn{2}{c|}{ Pessoa sem deficiência (PSD) } \\
\cline { 2 - 5 } D.T. & 0 & $0 \%$ & 0 & $0 \%$ \\
D. & 0 & $0 \%$ & 0 & $0 \%$ \\
I. & 2 & $13 \%$ & 0 & $0 \%$ \\
C. & 5 & $33 \%$ & 8 & $53 \%$ \\
C.T. & 8 & $54 \%$ & 7 & $47 \%$ \\
\hline TOTAL & 15 & $100 \%$ & 15 & $100 \%$ \\
\hline
\end{tabular}


O resultado foi positivo para ambos os grupos, não tendo nenhuma discordância quanto ao tema. Sendo que $100 \%$ das PSD concordaram com a assertiva.

\section{Discussão dos Resultados}

Analisando-se os resultados auferidos com a pesquisa de campo, pôde-se verificar que não houve diferença evidente entre a opinião apresentada pelas PCD e pelas PSD quanto à Qualidade de Vida no Trabalho. Isto porque as PCD obtiveram maior percentual de respostas positivas (concordo ou concordo totalmente) em dez assertivas. De forma similar, as PSD apresentam maior índice de concordância em outras dez assertivas. Cabe ainda ressaltar que quatro assertivas apresentaram empate entre as opiniões dadas por PCD e PSD.

Outra forma de analisar os resultados coletados diz respeito à tabulação de todas as respostas dos dois grupos pesquisados em relação aos pesos atribuídos nas respostas dadas à cada uma das 24 assertivas do instrumento de pesquisa. Assim, de forma geral, a opinião das pessoas com deficiência (PCD) foi levemente superior à opinião das pessoas sem deficiência (PSD). Os resultados obtidos foram demonstrados na Tabela 15:

Tabela 15 - Comparação geral dos resultados obtidos (PCD X PSD)

\begin{tabular}{|c|c|c|c|}
\hline \multicolumn{4}{|c|}{ PESSOAS COM DEFICIÊNCIA - PCD } \\
\hline ALTERNATIVA & QTDE. & PESO & PONTOS \\
\hline Discordo Totalmente & $\mathbf{1}$ & 1 & 1 \\
Discordo & $\mathbf{2 1}$ & 2 & 42 \\
Imparcial & $\mathbf{6 4}$ & 3 & 192 \\
Concordo & $\mathbf{1 9 7}$ & 4 & 788 \\
Concordo Totalmente & $\mathbf{7 7}$ & 5 & 385 \\
\hline \multicolumn{2}{|c}{} & Total & 1408 \\
\hline
\end{tabular}

\begin{tabular}{|c|c|c|c|}
\hline \multicolumn{4}{|c|}{ PESSOAS SEM DEFICIÊNCIA - PSD } \\
\hline ALTERNATIVA & QTDE. & PESO & PONTOS \\
\hline Discordo Totalmente & $\mathbf{1 1}$ & 1 & 11 \\
Discordo & $\mathbf{2 8}$ & 2 & 56 \\
Imparcial & $\mathbf{5 0}$ & 3 & 150 \\
Concordo & $\mathbf{2 0 6}$ & 4 & 824 \\
Concordo Totalmente & $\mathbf{6 5}$ & 5 & 325 \\
\hline \multicolumn{2}{|r}{} & Total & 1366 \\
\hline
\end{tabular}

Fonte: elaborado pelos autores. 
Pode-se observar que as PCD têm opinião levemente mais favorável em relação à Qualidade de Vida no Trabalho promovida pela empresa, tendo como resultado final 1408 pontos. Já as PSD obtiveram resultado de 1366 pontos. A variação encontrada entre os dois grupos de respondentes da pesquisa é inferior a 3\%. Ou seja, ao comparar a opinião das PCD e das PSD, não parece haver diferença estatisticamente significativa que indique representativa divergência de opinião entre os dois grupos analisados.

\section{Considerações Finais}

O respeito à diversidade nas empresas tem sido um tema cada vez mais discutido e inserido no contexto de negócios desenvolvidos por organizações modernas. Uma das ações desse âmbito é a inclusão de pessoas com deficiência (PCD) no quadro de funcionários, que também vêm ganhando força nos últimos anos principalmente devido à promulgação da 'lei de cotas' pelo Governo Federal em 1991.

A evolução na inclusão de PCDs no mercado de trabalho fez com que surgisse a preocupação de como estes colaboradores estão sendo incluídos em seus ambientes de trabalho. Embora a revisão da literatura tenha ressaltado a importância da qualidade de vida no trabalho (QVT) para todos os envolvidos na organização, este item passa a ser primordial ao se pensar nas particularidades vinculadas às PCD para o êxito desta inclusão.

Respondendo à pergunta de pesquisa, os resultados mostraram que as ações da empresa estudada visando a melhor inclusão de seus PCD surtiram efeito positivo, visto que a pesquisa de opinião identificou um posicionamento favorável por parte destes colaboradores aos temas que envolvem o desenvolvimento da QVT. Foi constatado também, considerandose a amostra avaliada, que empresa em questão desenvolve a QVT para todos os seus colaboradores, atendendo assim aos pontos indicados no modelo estudado. Essa conclusão advém da concordância expressada pela maioria dos colaboradores em todos os quesitos analisados.

Como resposta ao instrumento de pesquisa aplicado, pôde-se verificar que não houve significativa diferença nas respostas dos dois grupos analisados (PCD e PSD). Embora as PCD tenham apresentado resultados ligeiramente mais positivos, tais indicadores não se demonstraram significativamente superiores aos resultados das PSD. Das 24 assertivas constantes na pesquisa realizada, dez obtiveram maior pontuação para os respondentes PCD, outras dez apresentaram maior pontuação para os respondentes PSD, enquanto em quatro delas houve empate entre os dois conjuntos pesquisados.

Avaliando individualmente as questões em que a opinião de cada grupo foi maior, constatou-se que as PCD obtiveram opinião mais positiva quanto às questões relacionadas à satisfação com as atividades exercidas, liberdade em expressar sua opinião sobre seus próprios resultados, existência de um plano de carreira, segurança quanto à estabilidade no emprego, igualdade de oportunidade, bom relacionamento interpessoal e grupal, preservação da privacidade individual dos colaboradores, a liberdade de expressar sua opinião em todos os aspectos, balanceamento entre o trabalho e a vida particular dos colaboradores e, por fim, os colaboradores com deficiência se sentem mais responsáveis pelos produtos que a empresa produz e comercializa.

Já para as PSD foram constatadas opiniões mais positivas nas assertivas que envolvem a satisfação com a remuneração mediante as atividades exercidas, igualdade salarial dentro do setor e em comparação com as outras empresas, adequação da jornada de trabalho visando à preservação da saúde, a segurança no ambiente de trabalho, autonomia para execução das atividades em seu setor, oportunidade de crescimento profissional, cumprimento das leis e direitos trabalhistas, visão de uma boa imagem da empresa e, por fim ações de responsabilidade social da empresa. 
Os dois grupos tiveram a mesma opinião quanto às assertivas relacionadas à identificação da importância da atividade que executam para o resultado geral da empresa, competência para executar diversas tarefas, estímulo da empresa para o desenvolvimento do senso comunitário entre os colaboradores e, em complemento, clareza na divulgação das normas e rotinas da empresa.

Devido a isso, de forma geral, concluí-se que o investimento em ações que desenvolvam a QVT é relevante para garantir a satisfação do colaborador e mantê-lo integrado à organização. Não obstante, tal investimento também pode propiciar ganhos para a própria empresa por meio do aumento da produtividade e melhoria do clima organizacional.

Como limitação deste estudo, evidencia-se o fato desta pesquisa ter sido realizada a partir de observação em uma única empresa, com a aplicação de somente um modelo teórico para a realização da pesquisa de opinião. Outra limitação diz respeito à seleção de uma amostra por acessibilidade e conveniência dos pesquisadores, junto aos colaboradores com e sem deficiência da referida empresa.

Como sugestão para estudos futuros, indica-se a realização desta pesquisa em outros momentos nesta mesma empresa, para averiguar se houve evolução quanto às ações praticadas e a opinião dos colaboradores. Também é convidativo realizar estudo similar em empresas do mesmo ramo, para que se possam comparar os resultados e outra possibilidade é a execução deste estudo em empresas pertencentes ao ranking das 'Melhores Empresas para Trabalhar' definido pelas revistas Você S/A e Exame, visando verificar a relação da QVT com a posição da respectiva empresa no ranking. 


\section{Referências Bibliográficas}

ALVES, M.; GALEÃO-SILVA, L. A crítica da gestão da diversidade nas organizações. RAE - Revista de Administração de Empresas, São Paulo, v. 44, n. 3, p. 20-9, 2004.

BRASIL. Lei 8.213 - Decreto n. 129, de 22 de maio de 1991. Promulga a Convenção no 159, da Organização Internacional do Trabalho - OIT, sobre reabilitação profissional e emprego de pessoas deficientes. Diário Oficial da República Federativa do Brasil. Brasília: 22 maio 1991.

CENTERS, L.; CENTERS, R. Peer group attitudes toward the amputee child. Journal of Social Psychology, v. 61, p. 127-32, 1963.

CERVO, A. L.; BERVIAN, P. A. Metodologia científica. 5 ed. São Paulo: Pearson Prentice Hall, 2002.

COOPER, D. R.; SCHILNDLER, P. S. Métodos de pesquisa em administração. 7 ed. Porto Alegre: Bookman, 2003.

FARINA, A.; SHERMAN, J.; ALLEN, B. The role of the stigmatized person in affecting social relationships. Journal of Personality, v. 36, n. 2, p.169-82, 1968.

FLEURY, M. T. L. Gerenciando a diversidade cultural: experiência de empresas brasileiras. RAE - Revista de Administração de Empresas, São Paulo, v. 40, n. 3, p. 18-25, 2000.

FREITAS, M. E. O day-after das reestruturações: as irracionalidades e a coisificação do humano. RAE-Light, São Paulo, v. 6, n. 1, p. 5-7, jan./mar.1999.

GIL, A. C. Como elaborar projetos de pesquisa. 4 ed. São Paulo: Atlas, 2002.

HULAK, S. Psicossomática atual. Revista da Associação Brasileira de Medicina Psicossomática, v. 7, n. 1/2, jan./jun. 2003.

LIMONGI-FRANÇA, A. C. Qualidade de vida do trabalho \& administração. Programa de Educação Continuada em Administração para Executivos. São Paulo: FEA/USP, 2001.

MARCONI, M. A.; LAKATOS, E. M. Metodologia do trabalho científico: procedimentos básicos, pesquisa bibliográfica, projeto e relatório, publicações e trabalhos científicos. 6 ed. São Paulo: Atlas, 2001.

MORIN, E. Perception of justice, moral correctedness and employees'mental health. In: INTERNATIONAL CONGRESS OF APPLIED PSYCHOLOGY, 26, 2006, Procedings... Atenas: ICAP, 2006.

THOMPSON, V.; NOEL, J.; CAMPBELL, J. Stigmatization, discrimination, and mental health the impact of multiple identity status. American Journal of Orthopsychiatry, v. 74, n. 4, p. 529-44, 2004.

WALTON, R. E. Quality of working life: what is it? Sloan Management Review. v. 15, n. 1, p. 11-21, 1973.

WAILES, N.; MICHELSON, G. The transfer of management ideas to a western "periphery"; the case of corporate social responsability in Australia. International Studies of Management and Organization, v. 38, n. 4, p.100-18, 2008. 